\title{
Intoxicação por chumbo: conflitos ambientais na América do Sul e perspectiva sob a conservação de aves silvestres
}

\author{
Lead intoxication: environmental conflicts in South America and perspective under the \\ conservation of wild birds
}

Envenenamiento por plomo: conflictos ambientales en América del Sur y perspectiva sobre la conservación de aves silvestres

\section{Resumo}

O chumbo é um metal não essencial tóxico encontrado em diversas formas químicas, portanto, pode causar poluição atmosférica, terrestre e aquática. A principal fonte de chumbo se deve a ação antrópica, através da utilização de munições à base de chumbo, pesca, combustível, mineração, incineração de lixo e atividade industrial. O objetivo da presente revisão é demonstrar o conhecimento atual sobre as fontes de contaminação por chumbo, os sinais clínicos e lesões observadas em aves silvestres, diagnóstico e tratamento, impactos na biodiversidade e medidas de prevenção. Para a realização do estudo, foi utilizado pesquisa de artigos nas bases científicas PubMed, portal de periódicos CAPES, SciELO e Google Acadêmico, no recorte temporal de 2015 a 2020. A partir dos estudos elencados, observouse que os animais são frequentemente encontrados com alterações subclínicas, clínicas ou mortos em seus habitats naturais. A literatura afirma que os efeitos do chumbo no organismo variam de acordo com a dose, tempo de exposição e via de intoxicação, logo, as consequências na saúde das aves silvestres são inúmeras. Concluiu-se que a substituição do chumbo por outros componentes, alterações em normativas e conscientização sobre seu descarte, resultam em ganhos na conservação da natureza e melhoria da biodiversidade.

Palavras-chave: Biomarcadores; Efeitos deletérios; Medicina de aves; Metal pesado; Poluição.

\section{Abstract}

Lead is a non-essential toxic metal found in several chemical forms, therefore, it can cause air, land and water pollution. The main source of lead is due to anthropic action, through the use of lead-based ammunition, fishing, fuel, mining, waste incineration and industrial activity. The purpose of this review is to demonstrate current knowledge about the sources of lead contamination, the clinical signs and injuries observed in wild birds, diagnosis and 
treatment, impacts on biodiversity and prevention measures. In order to carry out the study, an article search was used in the scientific databases PubMed, the portal of CAPES, SciELO and Google Scholar journals, in the period from 2015 to 2020. From the studies listed, it was observed that animals are frequently found with subclinical or clinical changes, or even dead in their natural habitats. The literature states that the effects of lead in the body vary according to the dose, time of exposure and route of intoxication, therefore, the consequences on the health of wild birds are numerous. It was concluded that the replacement of lead by other components, changes in regulations and awareness of its disposal, result gains in nature conservation and improvement of biodiversity.

Keywords: Biomarker; Deleterious effects; Avian medicine; Heavy metal; Pollution.

\section{Resumen}

El plomo es un metal tóxico no esencial que se encuentra en varias formas químicas, por lo tanto, puede causar contaminación del aire, tierra y agua. La principal fuente de plomo se debe a la acción antrópica, mediante el uso de municiones a base de plomo, pesca, combustibles, minería, incineración de residuos y actividad industrial. El propósito de esta revisión es demostrar el conocimiento actual sobre las fuentes de contaminación por plomo, los signos clínicos y las lesiones observadas en las aves silvestres, el diagnóstico y tratamiento, los impactos en la biodiversidad y las medidas de prevención. Para la realización del estudio se utilizó la búsqueda de artículos en las bases científicas PubMed, portal de revistas CAPES, SciELO y Google Scholar, en el período de 2015 a 2020. De los estudios enumerados, se observó que los animales se encuentran con frecuencia con cambios subclínicos, clínicas o muertos en sus hábitats naturales. La literatura afirma que los efectos del plomo en el organismo varían según la dosis, el tiempo de exposición y la vía de intoxicación, por lo que las consecuencias sobre la salud de las aves silvestres son numerosas. Se llegó a la conclusión de que el reemplazo del plomo por otros componentes, los cambios en las regulaciones y la conciencia de su eliminación, dan como resultado ganancias en la conservación de la naturaleza y mejora de la biodiversidad.

Palabras clave: Biomarcadores; Efectos Nocivos; Medicina Avícola; Metales Pesados; Contaminación.

\section{Introdução}

A contaminação gerada pelos metais pesados fez com que os governantes, refletindo anseios da sociedade, repensassem as leis ambientais para minimizar a exposição aos contaminantes, seja por processos naturais ou atividades antrópicas (Alipour, et al., 2016). O arsênio (As), cádmio $(\mathrm{Cd})$, chumbo $(\mathrm{Pb})$, cobre $(\mathrm{Cu})$, cromo $(\mathrm{Cr})$, ferro $(\mathrm{Fe})$, manganês (Mn), mercúrio (Hg) e zinco ( $\mathrm{Zn}$ ) são metais que não se degradam no ambiente, dessa forma, acumulam-se ao longo da cadeia trófica permanecendo na natureza por mais tempo, com influência direta no habitat, na cadeia alimentar e em processos fisiológicos e migratórios de aves silvestres (Alipour, et al., 2016; Vizuete, et al., 2018).

O chumbo é um metal pesado que está onipresente no meio ambiente, sendo distribuído de três formas: chumbo metálico, sais de chumbo e chumbo orgânico (Assi, et al., 2016), entretanto, a atual fonte primária de contaminação por chumbo em aves silvestres se dá através da ingestão de projétil ou de seus fragmentos. Ao exposto, o $\mathrm{Pb}$ afeta a saúde de diferentes espécies de aves silvestres, sendo considerado um metal potencialmente tóxico, o qual ameaça a conservação da fauna (Plaza, et al., 2018).

$\mathrm{Na}$ América do Sul, Plaza et al. (2018) demonstraram presença de $\mathrm{Pb}$ em aves de 8 países, sendo estes, com maior ocorrência no Brasil (38,4\%), Argentina (33,3\%), Chile (7,7\%), Venezuela (7,7\%), Colômbia (5,1\%), Bolívia (2,6\%), Equador $(2,6 \%)$ e Peru (2,6\%). De todas as aves analisadas, foram identificadas 68 espécies, as quais de acordo com a "International Union for Conservation of Nature" (IUCN): uma espécie está criticamente em perigo, duas classificadas em perigo, sete estão quase ameaçadas, seis são vulneráveis e o restante encontra-se em baixo risco. Adiciona-se ainda que, dentre as espécies avaliadas neste continente, $50 \%$ eram aves aquáticas, 29,5\% eram aves de rapina e o restante eram de outros grupos, referente às ordens Tinamiformes, Columbiformes, Apodiformes e Passeriformes.

Algumas aves são mais suscetíveis a contaminação, e sem a intervenção terapêutica, podem morrer com uma única dose de chumbo em um curto período de tempo. Assim, é difícil a diferenciação entre exposição crônica e aguda, pois é dependente da quantidade que foi ingerida - uma única dose ou ingestão contínua de pequenas quantidades (Krone, 2018). A intoxicação por $\mathrm{Pb}$ pode resultar em três cenários: diminuição da população de presas; aumento da susceptibilidade à predação (por estarem comprometidas com os efeitos da intoxicação) e bioacumulação em predadores (Monzalvo-Santos, et al., 2016). 


\section{Metodologia}

O percurso metodológico desta revisão está respaldada por estudos publicados em periódicos indexados nas bases de dados PubMed, portal de periódicos CAPES, SciELO e Google Acadêmico, no recorte temporal de 2015 a 2020 e, segundo Pereira et al. (2018), o presente trabalho é do tipo qualitativo.

Para a busca de bibliografia de qualidade e reconhecida internacionalmente, foi utilizada a estratégia de pesquisa com uso de termos em inglês relacionados à intoxicação por chumbo em aves silvestres e impactos ambientais gerados por esse metal. Utilizou-se o filtro temporal dos cinco anos mais recentes e referências por algoritmo \{("Heavy Metal" OR "Wildlife" OR "Intoxication Birds" OR "Lead Poisoning Birds") AND (Environment Pollution OR "Lead pollution" AND "Conservation" AND "Wildlife Bird")\}. A soma das informações foi agrupada, apresentada e discutida em forma de revisão de literatura.

\section{Resultados e Discussão}

\subsection{Ingestão do Chumbo, Sinais Clínicos e Patologia}

Os animais terrestres podem absorver metais pesados, como o $\mathrm{Pb}$, por diversas vias como alimentação e respiração (Bjerregaard, et al., 2014). Dessa forma, a contaminação por chumbo pode afetar níveis tróficos maiores quando consomem carcaças contaminadas por este metal (Romero, et al., 2019; Hurtado, et al., 2020). O Pb pode ser ingerido acidentalmente por outras espécies de aves e também contaminar o meio ambiente (Pain, et al., 2019). Krone (2018) afirma que a ingestão oral de partículas de $\mathrm{Pb}$ é a via mais importante de contaminação em aves de rapina. O chumbo da munição está disponível para os predadores na carne de suas presas, seja como um projétil inteiro ou seus fragmentos. O predador pode se contaminar ao ingerir animais mortos ou restos de carcaça de animais provenientes da caça com munição de chumbo, ou quando animais são feridos, tornando-se vulneráveis a morte ou predação (Pain, et al., 2019).

A contaminação por chumbo, originária de caça, foi estudada em diversas espécies da Argentina por Plaza et al. (2018), foi verificado que a ingestão de $\mathrm{Pb}$ ocorre em aves aquáticas que habitam pântanos, e em aves necrófagas que vivem na região dos Andes. Já no continente Europeu, Monclús et al. (2020) relatam um aumento da concentração durante a temporada de caça nos países ao sul, que, embora exista proibição parcial do uso de $\mathrm{Pb}$ em munição de caça, ainda persiste a presença de $\mathrm{Pb}$ em rapinantes e os níveis de exposição superam o limiar subclínico.

Quando os fragmentos são ingeridos, eles acabam alojando-se no ventrículo gástrico para serem digeridos ou fundidos com penas, escamas, material vegetal ou outros. Devido ao peso e tamanho do metal ingerido, estes ficam presos nas dobras da mucosa, permanecendo até que sejam dissolvidos ou transportados até o intestino. O chumbo metálico é menos solúvel, desta forma o ácido clorídrico $(\mathrm{HCl})$ gástrico, produzido no ventrículo, cria uma camada de cloreto de chumbo na superfície, que passa por erosões e reconstruções. O cloreto de chumbo, torna-se solúvel na presença de $\mathrm{HCl}$ com a temperatura corporal. Além deste processo, há ligações de enzimas digestivas com a formação de sulfato de chumbo livre, o processo de dissolução continua até que as partículas de $\mathrm{Pb}$ sejam completamente dissolvidas com o auxílio de íons de bicarbonato, sais biliares e pancreatina (Fallon, et al., 2017; Krone, 2018). Espécies necrófagas como Gypaetus barbatus são mais suscetíveis à intoxicação, devido a elevada acidez estomacal que permite a dispersão das partículas de chumbo (Ganz, et al., 2018).

A absorção do $\mathrm{Pb}$ ocorre no duodeno, jejuno e íleo, sendo influenciada pela idade da ave, nutrição (ingestão de gordura, cálcio, ferro, vitamina D e vitamina E), solubilidade, tamanho da partícula, valor de $\mathrm{pH}$ e tempo de retenção (Krone, 2018). O Pb é absorvido e distribuído pela corrente sanguínea, em sua maior parte pelos eritrócitos (60-90\%, espécie dependente) e o restante é ligado às proteínas ou compostos de sulfidrila (Krone, 2018; Romero, et al., 2019).

Os efeitos subclínicos causados pelo chumbo podem afetar a atividade enzimática, levar ao estresse oxidativo e causar dano no DNA (Monclús, et al., 2020). O estudo de Espín et al. (2020) relaciona a exposição a este metal com níveis baixos de 
hematócrito. O Pb leva a inibição da enzima Ácido Delta Aminolevulínico Desidratase (ALAD) causando anemia por impedir a síntese do grupamento heme, o que acarreta em estimulação da produção de eritropoietina e maturação inadequada de eritrócitos (Krone, 2018).

Quando os animais são expostos a concentrações subletais de $\mathrm{Pb}$, podem ocorrer danos ao organismo em diferentes sistemas (Espín, et al., 2020), afetando o sistema imunológico, nervoso, renal, circulatório e reprodutor, com graves alterações nos seus processos fisiológicos (Vizuete, et al., 2018). Concentrações de chumbo no sangue maiores que 4 partes por milhão (ppm) são associadas a uma maior probabilidade de lesões cardiovasculares (Manning, et al., 2018).

Alguns dos sinais clínicos mais frequentemente relatados por diversos autores incluem anemia, emagrecimento, incoordenação, mudança de comportamento, asas caídas, paralisia do trato digestivo, convulsões, amaurose, vocalizações, letargia e dispneia, podendo afetar a aptidão, capacidade de voo e a performance do animal (Ecke, et al., 2017; Krone, 2018; Manning, et al., 2018; Romero, et al., 2019; Monclús, et al., 2020; Hurtado, et al., 2020). Além disso, os efeitos no sistema reprodutivo incluem alteração no tamanho dos ovos e interferência no desenvolvimento embrionário e da ninhada (MonzalvoSantos, et al., 2016; Yang, et al., 2020).

$\mathrm{Na}$ necropsia, é possível detectar alterações na morfologia hepática, aumento de vesícula biliar, ductos biliares dilatados, refluxo da bile para ventrículo, essas alterações não são patognomônicas, mas sim indicativas de intoxicação por chumbo (Krone, 2018). Em um estudo realizado por Isomursu et al. (2018), as alterações de vesícula biliar aumentada e bile viscosa com coloração verde escura foram encontradas em 97\% dos casos de intoxicação por chumbo. Romero et al. (2019) relacionam a ingestão de $\mathrm{Pb}$ com a coloração verde escura da mucosa do ventrículo de aves.

As lesões mais frequentes observadas no coração foram palidez miocárdica multifocal e arredondamento do ápice cardíaco. A necrose fibrinóide segmentar severa de artérias de pequeno a médio calibre é provavelmente a causa primária nas aves que foram intoxicadas por chumbo. Histologicamente, lesões dentro do coração podem representar um espectro de cronicidade referente a alterações de degeneração e necrose miocárdica (aguda), fibrose (subaguda a crônica) (Manning, et al., 2018) e pericardite fibrinosa (Krone, 2018).

Manning et al. (2018), encontraram na necropsia de Haliaeetus leucocephalus lesões simétricas bilaterais de aspecto esponjoso ou vacuolizado, presentes no tronco cerebral em 20,4\% das aves estudadas. Francisco et al. (2016) identificaram lesões em prosencéfalo, tronco cerebral e cerebelo, as quais foram relacionadas a alterações histológicas, tais como hemorragia intraparenquimatosa focal a multifocal e edema.

\subsection{Efeitos Deletérios do Chumbo no Organismo}

$\mathrm{A}$ exposição ao $\mathrm{Pb}$ no ambiente pode causar alterações nas populações de aves, afetando a eclodibilidade, o crescimento, sobrevivência, sucesso inicial dos filhotes, bem como problemas reprodutivos, tal qual a diminuição na produção de ovos devido baixa concentração plasmática de cálcio (Ding, et al., 2020). Yang et al. (2020) investigaram alterações no sistema reprodutor de machos de Passer montanus, expostos ao ambiente contaminado por metais pesados em diferentes províncias da China. Ao avaliarem a atividade de algumas enzimas no sêmen, foi encontrada redução na atividade da superóxido dismutase (SOD). Lactato desidrogenase (LDH) e Sorbitol desidrogenase (SDH) não apresentaram alterações. Porém, o hormônio Hormônio luteinizante (LH) e o hormônio folículo-estimulante (FSH), tiveram aumento. Apesar dessas alterações, o Passer montanus consegue compensar regulando o tamanho testicular, níveis de hormônio e qualidade do esperma.

Pruter et al. (2018), com intuito de identificar o efeito do chumbo na variedade de espécies de helmintos intestinais e a intensidade da infecção em patos selvagens (Anas platyrhynchos), verificaram que a riqueza de espécies de parasitas e intensidade de infestação foi significativamente menor em aves com níveis de $\mathrm{Pb}$ mais altos, sugerindo que tanto o hospedeiro 
quanto os parasitas respondem à exposição ao metal. No entanto, provavelmente também reflete nas diferenças de suscetibilidade dos diferentes helmintos ao $\mathrm{Pb}$. Nesse estudo a variedade de espécies de cestódeos e Acanthocephala foi particularmente afetada pela exposição ao $\mathrm{Pb}$. Concluindo que a intoxicação por $\mathrm{Pb}$ pode impactar negativamente no hospedeiro aviário e a diversidade do parasita em habitats aquáticos.

Além do exposto, o chumbo ainda pode impactar no desempenho de voo de Aquila chrysaetos, visto que concentrações subletais de chumbo no sangue de 25 ppb (partes por bilhão), referente ao peso úmido, foi associado à redução em $10 \%$ na altura do voo, já concentrações próximas de 43 ppb, a altura foi reduzida em $20 \%$, maiores concentrações diminuíram em 50\% (Ecke, et al., 2017).

Vizuete et al. (2018) documentam que concentrações abaixo de $2 \mu \mathrm{g} / \mathrm{g}$ de peso seco (dry weight $-d w$ ) podem sugerir que a ave encontra-se em um ambiente com exposição segura, sem risco toxicológico. Entretanto, demonstra que em tecidos de espécies de aves adultas, as quais vivem sob condições controladas de laboratório ou em áreas não contaminadas, a concentração de $\mathrm{Pb}$ foram de 2-15 $\mu \mathrm{g} / \mathrm{g}(d w)$ em tecido ósseo, 1-10 $\mu \mathrm{g} / \mathrm{g}$ em tecido renal e 0,5-5 $\mu \mathrm{g} / \mathrm{g}$ em tecido hepático. O estudo ainda inclui que a morte em patos selvagens é provocada com níveis entre 200-500 $\mu \mathrm{g} / \mathrm{g}(d w)$ no rim e 100-200 $\mu \mathrm{g} / \mathrm{g}$ no fígado.

\subsection{Amostras e Biomarcadores de Chumbo}

O delineamento da avifauna traduz papel importante na preservação e manutenção ambiental, uma vez que estes animais podem ser bioindicadores e detectar impactos ambientais oriundos, principalmente, de atividades humanas, nas comunidades naturais da ecologia. Além disso, as aves são passíveis a diversas variações nos ecossistemas, em virtude de características biológicas únicas (Batista, et al., 2020).

A dosagem de $\mathrm{Pb}$ no organismo do animal reflete as concentrações presentes no ambiente em que o mesmo vive, porém, especial atenção deve ser dada às aves migratórias que podem se contaminar em diferentes regiões (Ganz, et al., 2018). Dessa forma, a dosagem da concentração de $\mathrm{Pb}$ em tecidos, é utilizada como um bioindicador de contaminação (Burguer, et al., 2018; Romero, et al., 2019).

A utilização de amostras biológicas como sangue, osso, penas, fígado, rim, músculo, cérebro, gônadas, ovos e fezes são usados para avaliar contaminação por chumbo. Há algumas particularidades, como na amostra de fezes, que não são habitualmente utilizadas para diagnóstico de contaminação por $\mathrm{Pb}$, pois não são úteis como indicadores de bioacumulação (Espín, et al., 2016).

Os maiores valores de $\mathrm{Pb}$ encontram-se em ossos, rins e fígado (Binkowski, et al., 2016; Espín, et al., 2020), tendo menores concentrações em sistema nervoso central e sangue, bem como em tecido muscular, visto que, dos três tecidos, este apresenta as menores concentrações em aves. Ainda, descreve-se que em algumas espécies de aves rapinantes, existe uma diferenciação nos valores de concentração no tecido renal e hepático (Krone, 2018).

A concentração de $\mathrm{Pb}$ no sangue aumenta dentro de 24 horas após absorção, com meia-vida plasmática de semanas a meses, já em tecidos moles, a meia-vida é de 1 a 3 meses. Por fim deposita-se na matriz óssea ocupando o local do cálcio, interferindo na remodelação óssea e na síntese de ovos (Krone, 2018). A dosagem de Pb no sangue demonstra uma exposição recente do indivíduo, em média de 30 dias (Binkowski, et al., 2016; Sriram, et al., 2018; Kruger \& Amar, 2018).

Um estudo realizado na Nova Zelândia por Sriram et al. (2018) dosou a concentração de Pb no sangue em indivíduos de Nestor meridionalis septentrionalis de áreas urbanas. Das aves analisadas, 43,2\% apresentaram níveis de exposição ao chumbo, sendo que $87 \%$ possuíam concentração de $\mathrm{Pb}$ abaixo de $20 \mu \mathrm{g} / \mathrm{dL}$. Apenas uma ave analisada apresentava sinais clínicos de intoxicação, esse indivíduo teve concentração sanguínea de $50,7 \mu \mathrm{g} / \mathrm{dL}$. 
As penas e ossos são considerados os melhores tecidos para avaliar a contaminação e exposição crônica por $\mathrm{Pb}$ (Monzalvo-Santos, et al., 2016; Kruger \& Amar, 2018). Monzalvo-Santos et al. (2016) analisaram as concentrações de Pb em amostras de solo e penas de passeriformes residentes em áreas expostas e não expostas à mineração no México. As concentrações de chumbo nas penas variaram de acordo com as concentrações no solo, sendo mais elevadas em áreas próximas à mineração.

Seone et al. (2018), exploram o uso de diferentes porções das penas de Strix aluco como biomonitoramento ambiental de metais, tais quais, $\mathrm{As}, \mathrm{Cd}$ e $\mathrm{Pb}$. A raque da pena primária foi subdividida em dez partes ( $\mathrm{S} 1$ a S10, sendo a última mais distante ao corpo), a qual a concentração máxima de $\mathrm{Pb}$ permaneceu constante, entretanto, em termo de secções, as maiores concentrações permaneceram entre as partes S6-S8, levando em consideração o coeficiente de determinação com relação às quantidades de metal de todas as raques de uma asa. Com a riqueza destes elementos na raque, há uma representação de acúmulo por via endógena, nisto, há de se considerar o padrão de muda em S. aluco. Embora essa não seja sincrônica, ela é completa. Subsequente, a concentração de metais é mais elevada em penas que são substituídas mais cedo.

Com a utilização de penas, como amostra biológica de aves marinhas, os níveis de $\mathrm{Pb}$ apresentaram variações expressivas de acordo com suas espécies e regiões encontradas. Rissa tridactyla (Alasca) e Larus ridibundus (Polônia) apresentaram variação de 0,707 $\pm 0,131$ a 10,40 $\pm 5,36 \mu \mathrm{g} / \mathrm{g}$, respectivamente. A comparação entre gaivotas em geral, Sterna hirundo, Larus argentatus no porto de Nova York e Leucophaeus pipixcan em Midwest, mostrou que os níveis de $\mathrm{Pb}$ em penas de gaivotas e andorinhas, de acordo a 31 estudos, variaram de 0,1 a 4,3 $\mu \mathrm{g} / \mathrm{g}$ (mediana: 1,3 $\mu \mathrm{g} / \mathrm{g}$ ) (Vizuete, et al., 2018).

Entretanto, Grðz et al. (2015), mensuraram a contaminação por metais pesados de Cygnus olor no ambiente aquático de Keszthely Bay, Lago Balaton, Hungria, e compararam as amostras de penas de contorno com 8 diferentes espécies de plantas que fazem parte da dieta dos cisnes. Os resultados demonstram que as concentrações de metais pesados foram maiores nas plantas do que na plumagem.

A utilização da espécie Gypaetus barbatus em Kruger \& Amar (2018) revelou que a concentração de $\mathrm{Pb}$ no tecido ósseo estava entre 8,34 - 11,79 $\mu \mathrm{g} / \mathrm{g}$ sugerindo exposição ao $\mathrm{Pb}$, sendo que dois indivíduos juvenis apresentaram níveis de exposição patológica $(19,96$ e 20,92 $\mu \mathrm{g} / \mathrm{g})$.

O diagnóstico de intoxicação pode ser feito através da dosagem de $\mathrm{Pb}$ em rins e fígado, com nível maior que $5 \mathrm{mg} / \mathrm{kg}$. A concentração média de chumbo nestes tecidos, na espécie Haliaeetus albicilla, foram de 9,2 mg/kg e 18,7 mg/kg, na devida ordem (Isomursu, et al., 2018). Ao empregar gaivotas como referência, Vizuete et al. (2018), dosaram o chumbo nas espécies L. argentatus da Itália $e$ L. ridibundus da Polônia, e obtiveram resultados em tecido hepático de $<0,1$ a $8,91 \pm 1.87 \mu \mathrm{g} / \mathrm{g}$, respectivamente. Em contrapartida, em tecido renal a variação foi ainda maior, revelando variações de $<0,1$ a $30,96 \pm 22,81$ $\mu \mathrm{g} / \mathrm{g}$.

A análise de redundância (RDA), realizada por Kitowski et al. (2016) pode ser utilizada para relacionar concentrações de metais pesados e hábitos alimentares de diversas espécies, dentre elas, o Buteo buteo, o qual apresentou níveis relevantes de concentração de chumbo $\geq 6 \mathrm{mg} / \mathrm{kg}$ peso seco $(d w)$ e apenas um exemplar apresentou $>15 \mathrm{mg} / \mathrm{kg}$ no fígado, correspondente ao consumo de pequenos e médios mamíferos e carcaça. Segundo Artin et al. (2017) em estudo realizado com Haliaeetus leucocephalus, a média encontrada de concentração de $\mathrm{Pb}$ no tecido hepático foi de $81,01 \mu \mathrm{g} / \mathrm{g}$, esse valor pode alterar-se, pois munições à base de $\mathrm{Pb}$ ou partículas das mesmas em seus estômagos, podem fazer com que a concentração no tecido hepático fique aumentada.

Ao avaliar a idade e acúmulo deste metal em aves marinhas, foi encontrado Puffinus gravis adultas no litoral brasileiro, e esta exibiu elevados níveis hepáticos de Pb comparado aos exemplares jovens (Vizuete, et al., 2018). Bem como no estudo realizado por Ganz et al. (2018), com Milvus milvus, demonstrou maior acúmulo de $\mathrm{Pb}$ nas aves adultas. No entanto, os autores afirmam que existem dados controversos, tal qual o estudo de Burguer et al. (2018) onde os níveis de chumbo 
encontrado em Calidris pusilla, foram maiores em aves mais jovens, até 2 anos, do que em aves adultas, acima de 2 anos. Isso pode ser atribuído à não ocorrência de migração por se tratar de animais muito jovens e também pela ausência na troca de penas.

A consequência natural é o acúmulo com a idade, mas quando a absorção das aves ultrapassa o poder de excreção desses elementos inorgânicos, deve-se sempre observar espécie, órgão e ambiente, tornando complexa a análise entre níveis de $\mathrm{Pb}$ e idade. Em relação ao sexo dos animais, não houve relatos de diferenças significativas, com exceção do Rynchops niger, espécie com acentuado dimorfismo sexual, em que verificou-se maiores concentrações de chumbo nas fêmeas em comparação aos machos da mesma espécie (Vizuete, et al., 2018).

\subsection{Diagnóstico e Tratamento}

Os sinais clínicos e achados anatomopatológicos são inespecíficos da intoxicação por chumbo, porém sinais neurológicos (Monclús, et al., 2020) e alterações na vesícula biliar (Isomursu, et al., 2018) são sugestivos de intoxicação. De acordo com Fallon et al. (2017), o diagnóstico nos casos de intoxicação por Pb pode ser feito por diferentes métodos. O exame radiológico pode ser utilizado para identificar fragmentos de metais no trato gastrointestinal, a fim de confirmar a presença de $\mathrm{Pb}$ (Pain, et al., 2019), para isso, deve ser feito o uso da radiologia combinada à dosagens sanguíneas do metal, tendo em vista que outros metais apresentam densidade semelhante ao chumbo (Fallon, et al., 2017).

Para dosagem sanguínea de chumbo diversos autores apontam concentrações diferentes em casos de intoxicação entre as espécies de aves. Fallon et al. (2017) classificam, em rapinantes, concentrações $\leq 40 \mu \mathrm{g} / \mathrm{dL}$ como baixa, $40-60 \mu \mathrm{g} / \mathrm{dL}$ como elevada e $\geq 60 \mu \mathrm{g} / \mathrm{dL}$ como tóxicas. Enquanto Kruger e Amar (2018) sugerem que concentrações acima de $40 \mu \mathrm{g} / \mathrm{dL}$ já demonstram sinais de intoxicações em rapinantes e abutres. Em um estudo com Nestor meridionalis septentrionalis foi encontrado concentração de chumbo de 50,7 $\mu \mathrm{g} / \mathrm{dL}$ em um animal com sinais de intoxicação, enquanto a maioria dos expostos não apresentavam sinais clínicos tiveram dosagem de Pb abaixo de $20 \mu \mathrm{g} / \mathrm{dL}$ (Sriram, et al., 2018). Em tecidos hepáticos as concentrações de $\mathrm{Pb}$ atingem níveis de intoxicação subclínica de 2,0 a 6,0 ppm, intoxicação clínica de 6,0 a 15,0 ppm e envenenamento clínico severo em concentrações acima de 15,0 ppm (Kanstrup, et al., 2019; Herring \& Eagles-Smith, 2017).

Para verificação de lesões no sistema nervoso, é possível aplicar ressonância magnética para identificar áreas neurais comprometidas, como espongiose em substância branca de cérebro, cerebelo e medula oblonga (Krone, 2018). No entanto, essas alterações não são patognomônicas da intoxicação, mas esses achados podem sugerir provável intoxicação.

Algumas terapias quelantes estão descritas na literatura, como o uso do ácido etilenodiamina-tetraacético de cálcio (CaEDTA) e do ácido dimercaptosuccínico (DMSA) (Fallon, et al., 2017; Manning, et al., 2018). Para que a terapia com quelantes seja eficaz é necessária a remoção dos fragmentos de chumbo do trato gastrointestinal, que pode ser feita através de endoscopia, proventriculotomia, lavagem gástrica, ou uso de laxantes e catárticos que auxiliam na eliminação (Fallon, et al., 2016). A terapia de quelação foi associada a menores concentrações de chumbo nos tecidos, cerca de 13,6 ppm, quando comparado aos animais que não foram submetidos ao tratamento, 22,9 ppm. Esta diferença também foi aparente na concentração média de $\mathrm{Pb}$ nos rins, 1,8 ppm em aves tratadas e 7,4 ppm para aves não tratadas (Manning, et al., 2018).

\subsection{Poluição Gerada por Atividades Antrópicas}

Segundo Bjerregaard et al. (2014), a mobilização e emissão de metais pesados é proveniente de diversas atividades humanas, de forma direta e indireta (alterando o ambiente e mobilizando metais que antes encontravam-se de forma estável no ambiente). Essa exposição pode derivar de diversas vertentes como: munição de caça, combustível, pesca, mineração, indústria, zonas marinhas costeiras poluídas e esporte com barco a motor (Plaza, et al., 2018; Monclús, et al., 2020). O chumbo 
é persistente no ambiente por longos períodos, então mesmo emissões antigas podem ser fontes de contaminação (Sriram, et al., 2018).

A incineração de lixo, queima de combustível fóssil e atividade siderúrgica são as principais fontes de emissão direta de chumbo na atmosfera através de erosão de materiais metálicos (de calhas por exemplo) e por esgoto doméstico; enquanto mineração e outras atividades industriais constituem as principais fontes de contaminação de águas (Bjerregaard, et al., 2014).

Quando o lixo comercial é descartado, este é agregado aos resíduos domésticos, o qual se torna uma fonte atrativa de alimentação para aves marinhas, moluscos e outros invertebrados que podem ser identificados nestes locais. Além da ação antropogênica discorrida, o estudo também inclui exposição de $\mathrm{Pb}$ em filhotes de albatrozes em Sand Island e albatrozes adultos em Midway Atoll nos Estados Unidos da América (EUA), associados às obras nestes locais, com a ingestão de lascas de tinta (Vizuete, et al., 2018).

Os hábitos alimentares adotados por aves silvestres assumem um papel importante nos depósitos deste metal (Vizuete, et al., 2018). Em estudo realizado por Turzańska-Pietras et al. (2017), o conteúdo de oligoelementos nos excrementos de filhotes de Sylvia communis de ninhos localizados próximos a um depósito de metais, foi em média 3,9 vezes maior do que os níveis do solo próximo ao aterro, o que sugere o acúmulo de metais em alimentos consumidos pelas aves.

O chumbo pode ser exposto indiretamente no ambiente através do uso de munições que contém esse metal em sua composição, e por meio de eventos como chuvas ácidas, que, por serem ricas em cátions, mobilizam metais pesados presentes de forma estável no ambiente, podendo levar inclusive à acidificação de corpos d'água (Bjerregaard, et al., 2014). Romano et al. (2016) afirmam que a contaminação de $\mathrm{Pb}$ permaneça ou aumente devido à caça (esportiva e de subsistência), especialmente devido à bioacumulação com falta de biodegradação deste metal tóxico.

Isomursu et al. (2018), Plaza et al. (2018) e Pain et al. (2019) identificaram que os maiores índices de intoxicação em aves foram relacionados com as épocas de maior intensidade de caça nas regiões estudadas, sugerindo que projéteis são a principal fonte de contaminação por chumbo. Em estudo de Romero et al. (2019), os autores demonstraram diferenças na concentração de $\mathrm{Pb}$ em área urbana, periurbana e rural, levando em consideração a intensidade de caça. Neste, afirmam que aves de áreas periurbanas também apresentam níveis elevados de $\mathrm{Pb}$. Os níveis de chumbo encontrados em aves com fragmentos de projéteis no trato digestório são maiores do que naquelas sem indícios de consumo, porém os autores sugerem baixa exposição ambiental por ingestão de projéteis e que, em algumas espécies, a exposição é proveniente de áreas urbanas.

Durante a caça de alces na Fenoscândia, a cada ano, cerca de $215 \mathrm{~kg}$ de chumbo provenientes de munição é encontrado em vísceras e carcaças deixadas no ambiente, esse valor é correspondente a mais de 100.000 doses letais para águias (Stokke, et al., 2017). As competições de caça de lebre da montanha (Lepus timidus), perdiz da floresta (Tetrao urogallus, Lyrurus tetrix, Lagopus lagopus e Bonasa bonasia), corça (Capreolus capreolus) e raposa vermelha (Vulpes vulpes), quando ocorrem fora da temporada, prejudicam e aumentam ainda mais a exposição de Aquila chrysaetos e outros animais selvagens ao chumbo (Ecke, et al., 2017).

Regulamentações para mitigar essas fontes de chumbo foram implementadas globalmente, deste modo, é esperado uma redução na contaminação produzida. Nos últimos 25 a 30 anos, as políticas ambientais aumentaram em diferentes países da América do Sul, porém, as atividades de mineração permanecem como fonte importante de contaminação. O mesmo ocorre na indústria petroquímica e fabricantes de baterias, então, ressalva-se que, apesar de regulamentações atuais, não há como acabar com algumas fontes de chumbo e depósitos no ambiente (Plaza, et al., 2018).

Na América do Sul há normativas sobre a utilização do metal na composição de tintas e combustíveis que buscam diminuir a contaminação ambiental (Plaza, et al., 2018). Em estudo realizado na Europa já foi demonstrado que as concentrações de $\mathrm{Pb}$ não tem aumentado em relação ao ambiente, devido a regulamentações que proíbem a presença de metais pesados em combustíveis (Helander, et al., 2019). Entretanto, existem poucos regulamentos que limitam o uso do chumbo em 
munição em todo mundo, inclusive na América do Sul (Plaza, et al., 2018). A escassez de normas torna-se lamentável, pois, o $\mathrm{Pb}$ derivado de munições é um grande problema ambiental que tem como uma solução o uso de cartuchos alternativos, como foi instaurado na Dinamarca, o que diminuiria significativamente as mortes por intoxicação, aumentando o bem-estar da vida selvagem (Arnemo, et al., 2016; Pain, et al., 2019).

Um estudo realizado por Kanstrup et al. (2019) comprova que a redução progressiva do chumbo em munição para caça, desde 1986, gerou redução dos níveis de $\mathrm{Pb}$ em aves de rapina dinamarquesas, visto que de todas as amostras de tecido hepático coletadas, apenas cinco excederam concentrações de 1 ppm e uma excedeu o critério para intoxicação subclínica de 2 ppm. Binkowski et al. (2016) apresentaram resultados positivos para chumbo no sangue com concentração de $0,241 \mu \mathrm{g} / \mathrm{g} \mathrm{de}$ peso úmido, entretanto as amostras indicam que o $\mathrm{Pb}$ não é proveniente de projéteis, devido à grande quantidade de outros minérios. A distinção proveniente de munição, contaminação do solo e migração das aves torna impossível saber a procedência do $\mathrm{Pb}$ presente.

Desta forma, a compilação destas informações acerca de áreas com alto potencial de contaminação por $\mathrm{Pb}$ e produção de mapas de risco, sugerem uma importância para confecção destes com áreas prioritárias para a vigilância de toxicidade do metal (Plaza, et al., 2018).

\section{Considerações Finais}

O chumbo ainda é amplamente utilizado pela indústria devido a facilidade e durabilidade que o metal apresenta, todavia, há uma tendência à redução no uso deste material e substituição por materiais alternativos, os quais apresentam menor nível de toxicidade e boa funcionalidade.

No Brasil, o chumbo procedente de munição ainda é uma fonte subestimada e pouco regulamentada, visto que, a instrução normativa de $n^{\circ} 12$ (IBAMA, 2019) dispõe acerca da perseguição, abate e captura, entretanto observa-se uma carência no que diz respeito à eliminação da carcaça por exemplo de javali (Sus scrofa), espécie exótica invasora e legalizada para controle e abate com armas brancas e de fogo. Dessa forma, há de se reavaliar as informações declaradas nesta normativa e no momento da inscrição do Cadastro Técnico Federal, em relação ao controle populacional da espécie, a qual aponta ser uma atividade potencialmente poluidora em todo país.

A complexidade de mensuração e interpretação de análises está vinculada a ausência de valores de referência padronizados. A falta dessas informações dificulta a confirmação do diagnóstico, e, por consequência, gera atraso no tratamento desses animais. No presente momento, a confirmação desses resultados varia de acordo com as informações provenientes de diferentes fontes, as quais não estão uniformizadas.

Visto fundamentos, enfatiza-se que as concentrações de $\mathrm{Pb}$ representam uma contaminação externa, e deve-se ponderar as adversidades causadas por este metal na dieta, dado que a ingestão acidental de chumbo está inclusa nos hábitos alimentares de diversas aves, especialmente as necrófagas. Ademais, o ambiente que este animal está inserido também deve ser avaliado, pois, as ações antrópicas ocasionadas por descarte incorreto desse metal, bem como, exploração em áreas de mineração, interferem diretamente na saúde e conservação geográfica de aves silvestres.

As informações encontradas na literatura consultada, demonstram lacunas no que se refere a padronização de metodologias de mensuração e qual a melhor amostra biológica a ser utilizada. Estudos futuros de mapeamento de regiões com elevado potencial de contaminação devem ser elaborados, auxiliando com isso, a regulamentação de estratégias que minimizem a contaminação ambiental pelo $\mathrm{Pb}$. 


\section{Referências}

Alipour, H., Solgi, E., \& Majnouni F. (2016). Concentrations of Heavy Metals in tissues of the Mallard Anas platyrhynchos in Kanibarazan, northwestern Iran.

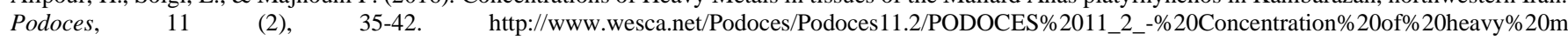
etals\%20in\%20Mallard.pdf

Arnemo, J. M., Andersen, O., Stokke, S., Thomas, V. G., Krone, O., Pain, D. J., \& Mateo, R. (2016). Health and Environmental Risks from Lead-based Ammunition: science versus socio-politics. Ecohealth, 13 (4), 618-622. 10.1007/s10393-016-1177-X

Artin, P. A., Hughes, K. D., Campbell, G. D., \& Shutt, J. L. (2017). Metals and Organohalogen Contaminants in Bald Eagles (Haliaeetus leucocephalus) from Ontario, 1991-2008. Archives Of Environmental Contamination And Toxicology, 74 (2), 305-317. 10.1007/s00244-017-0479-5

Assi, M. A., Hezmee, M. N. M., Haron, A. W., Sabri, M. Y. M., \& Rajion, M. A. (2016). The detrimental effects of lead on human and animal health. Veterinary World, 9 (6), 660-671. 10.14202/vetworld.2016.660-671

Batista, M. S., Brum, B. R., Hurtado, T. C., \& Ignácio, Áurea R. A. (2020). Pesticidas em aves insetívoras: uma análise quantitativa sobre o panorama de contaminação. Research, Society and Development, 9 (9), e142996483. https://doi.org/10.33448/rsd-v9i9.6483

Binkowskl, L., Meissner, W., Trzeciak, M., Izevbekhai, K., \& Barker, J. (2016). Lead isotope ratio measurements as indicators for the source of lead poisoning in Mute swans (Cygnus olor) wintering in Puck Bay (northern Poland). Chemosphere, 164, 436-442. 10.1016/j.chemosphere.2016.08.120

Bjerregaard, P., Andersen, C. B. I., \& Andersen, O. (2014). Ecotoxicology of Metals: Sources, Transport, and Effects on the Ecosystem. In Nordberg, G. F.; Fowler, B. A.; Nordberg, M (4a ed.). Handbook on the Toxicology of Metals (pp. 426 - 459). Amsterdam: Elsevier.

Burger, J., Mizrahi, D., Tsipoura, N., Jeitner, C., \& Gochfeld, M. (2018). Mercury, Lead, Cadmium, Cobalt, Arsenic and Selenium in the Blood of Semipalmated Sandpipers (Calidris pusilla) from Suriname, South America: age-related differences in wintering site and comparisons with a stopover site in new jersey, USA. Toxics, 6 (2), 6-27. 10.3390/toxics6020027

Ding, J., Yang, W., Wang, S., Zhang, H., Yang, Y., Bao, X., \& Zhang, Y. (2020). Effects of environmental metal pollution on reproduction of a free-living resident songbird, the tree sparrow (Passer montanus). Science Of The Total Environment, 721, 137674. 10.1016/j.scitotenv.2020.137674

Ecke, F., Singh, N. J., Arnemo, J. M., Bignert, A., Helander, B., Berglund, A. M. M., Borg, H., Bröjer, C., Holm, K., Lanzone, M., Miller, T., Nordström, A., Räikkönen, J., Rodushkin, I., Agren, E., \& Hörnfeldt, B. (2017). Sublethal Lead Exposure Alters Movement Behavior in Free-Ranging Golden Eagles. Environmental Science \& Technology, 51 (10), 5729-5736. 10.1021/acs.est.6b06024

Espín, S., García-Fernández, A. J., Herzke, D., Shore, R. F., van Hattum, B., Martínez-López E., Coeurdassier, M., Eulaers, I., Fritsch, C., Gómez-Ramírez, P., Jaspers, V. L. B., Krone, O., Duke, G., Helander, B., Mateo, R., Movalli, P., Sonne, C., \& van den Brink, N. W. (2016). Tracking pan-continental trends in environmental contamination using sentinel raptors-what types of samples should we use? Ecotoxicology, 25 (4), 777-801. 10.1007/s10646-016-1636-8

Espín, S., Sánchez-Virosta, P., Zamora-Marín, J. M., León-Ortega, M., Jiménez, P., Zumbado, M., Luzardo, O. P., Eeva, T., \& García-Fernández, A. J. (2020). Toxic elements in blood of red-necked nightjars (Caprimulgus ruficollis) inhabiting differently polluted environments. Environmental Pollution, $262,114334$. 10.1016/j.envpol.2020.114334

Fallon, J. A., Redig, P., Miller, T. A., Lanzone, M., \& Katzner, T. (2017). Guidelines for evaluation and treatment of lead poisoning of wild raptors. Wildlife Society Bulletin, 41 (2), 205-211. 10.1002/wsb.762

Francisco, O. N., Feeney, D., Armién, A.G., Wuenschmann, A., \& Redig, P. T. (2016). Correlation of brain Magnetic Resonance Imaging of spontaneously lead poisoned bald eagles (Haliaeetus leucocephalus) with histological lesions: a pilot study. Research In Veterinary Science, 105 (1), 236-242. 10.1016/j.rvsc.2016.02.010

Ganz, K., Jenni, L., Madry, M. M., Kraemer, T., Jenny, H., \& Jenny, D. (2018). Acute and Chronic Lead Exposure in Four Avian Scavenger Species in Switzerland. Archives Of Environmental Contamination And Toxicology, 75 (4), 566-575. 10.1007/s00244-018-0561-7

Grúz, A., Szemerédy. G., Kormos, E., Budai, P., Majoros, S., Tompai, E., \& Lehel, J. (2015). Monitoring of heavy metal burden in mute swan (Cygnus olor). Environmental Science And Pollution Research, 22 (20), 15903-15909. 10.1007/s11356-015-4809-8

Helander, B., Sundbom, M., Runkel, A. A., \& Bignert, A. (2019). Temporal Changes in Concentrations of Lead and Other Trace Metals in Free-Ranging Eurasian Eagle Owls Bubo bubo in Sweden. Archives Of Environmental Contamination And Toxicology, 77 (3), 377-389. 10.1007/s00244-019-00654-5

Herring, G., \& Eagles-Smith, C. A. (2017). Characterizing Golden Eagle Risk to Lead and Anticoagulant Rodenticide Exposure: a review. Journal Of Raptor Research, 51 (3), 273-292. 10.3356/JRR-16-19.1

Hurtado, T. C., Brum, B. R., Batista, M. S., D’ Ávila, R. dos S., \& Ignácio, Áurea R. A. (2020). Estudo quantitativo temporal sobre a contaminação de aves aquáticas por metais. Research, Society and Development, 9 (8), e993986710. 10.33448/rsd-v9i8.6710

Instituto Brasileiro do Meio Ambiente e dos Recursos Naturais Renováveis (IBAMA). (2019). Instrução normativa $\mathrm{n}^{\circ} 12$, de 25 de março de 2019. Aprova a Estrutura Regimental do Ibama. Brasília, 29-33. https://www.in.gov.br/materia/-/asset_publisher/Kujrw0TZC2Mb/content/id/70006375/do1-2019-04-04instrucao-normativa-n-12-de-25-de-marco-de-2019-70006233

Isomursu, M., Koivusaari, J., Stjernberg, T., Hirvelä-Koski, V., \& Venäläinen, E. R. (2018). Lead poisoning and other human-related factors cause significant mortality in white-tailed eagles. Ambio, 47 (8), 858-868. 10.1007/s13280-018-1052-9

Kanstrup, N., Chriél, M., Dietz, R., Søndergaard, J., Balsby, T. J. S., \& Sonne, C. (2019). Lead and Other Trace Elements in Danish Birds of Prey. Archives Of Environmental Contamination And Toxicology, 77 (3), 359-367. 10.1007/s00244-019-00646-5 
Kitowski, I., Sujak, A., Wiącek, D., Strobel, W., Komosa, A., \& Stobiński, M. (2020). Heavy metals in livers of raptors from Eastern Poland - the importance of diet composition. Belgian Journal Of Zoology, 146 (1), 3-13. 10.26496/bjz.2016.34

Krone, O. (2018). Birds of Prey: Biology and Conservation in the XXI Century (1a ed). Berlim: Springer.

Krüger S. C., \& Amar, A. (2018). Lead Exposure in the Critically Endangered Bearded Vulture (Gypaetus barbatus) Population in Southern Africa. Journal Of Raptor Research, 52 (4), 491-499. 10.3356/jrr-17-86.1

Manning, L. K., Wünschmann, A., Armién, A. G., Willette, M., MacAulay, K., Bender, J. B., Buchweitz, J. P., \& Redig P. (2018). Lead Intoxication in FreeRanging Bald Eagles (Haliaeetus leucocephalus). Veterinary Pathology, 56 (2), 289-299. 10.1177/0300985818813099

Monclús, L., Shore, R. F. \& Krone, O. (2020). Lead contamination in raptors in Europe: a systematic review and meta-analysis. Science Of The Total Environment, 748, 141437. 10.1016/j.scitotenv.2020.141437

Monzalvo-Santos, K., Alfaro-De la Torre, M. C., Chapa-Vargas, L., Castro-Larragoitia, J., \& Rodríguez-Estrella, R. (2016). Arsenic and lead contamination in soil and in feathers of three resident passerine species in a semi-arid mining region of the Mexican plateau. Journal of Environmental Science and Health, Part A, 51 (10), 825-832. 10.1080/10934529.2016.1181451

Pain, D. J., Mateo, R., \& Green, R. E. (2019). Effects of lead from ammunition on birds and other wildlife: A review and update. Ambio, 48 (9), 935-953. $10.1007 / \mathrm{s} 13280-019-01159-0$

Pereira, A. S., et al. (2018). Metodologia da pesquisa científica. [e-book]. Santa Maria. Ed. UAB/NTE/UFSM. Recuperado de https://repositorio.ufsm.br/bitstream/handle/1/15824/Lic_Computacao_Metodologia-Pesquisa-Cientifica.pdf?sequence=1 .

Plaża. P., Uhart, M., Caselli, A., Wiemeyer, G., \& Lambertucci, S. A. (2018). A review of lead contamination in South American birds: the need for more research and policy changes. Perspectives In Ecology And Conservation, 16 (4), 201-207. 10.1016/j.pecon.2018.08.001

Pruter, H., Franz, M., Auls, S., Czirják, G. Á., Greben, O., Greenwood, A. D., Lisitsyna O., Syrota, Y., Jilji, S., Krone, O. (2018). Chronic lead intoxication decreases intestinal helminth species richness and infection intensity in mallards (Anas platyrhynchos). Science Of The Total Environment, 644, 151-160. 10.1016/j.scitotenv.2018.06.297

Romano, M., Ferreyra, H., Ferreyroa, G., Molina, F. V., Caselli, A., Barberis, I., Beldoménico, P., \& Uhart, M. (2016). Lead pollution from waterfowl hunting in wetlands and rice fields in Argentina. Science Of The Total Environment, 545, 104-113. 10.1016/j.scitotenv.2015.12.075

Seoane, R. G., Río, Z. V., Ocaña, A. C., Escribano, J. A. F., \& Viñas, J. R. A. (2018). Selection of tawny owl (Strix aluco) flight feather shaft for biomonitoring As, Cd and Pb pollution. Environmental Science And Pollution Research, 25 (14), 14271-14276. 10.1007/s11356-018-1477-5

Sriram, A., Roe, W., Booth, M., \& Gartrell, B. (2018). Lead exposure in an urban, free-ranging parrot: investigating prevalence, effect and source attribution using stable isotope analysis. Science Of The Total Environment, 634, 109-115. 10.1016/j.scitotenv.2018.03.267

Stokke, S., Brainerd, S., \& Arnemo, J. M. (2017). Metal deposition of copper and lead bullets in moose harvested in Fennoscandia. Wildlife Society Bulletin, 41 (1), 98-106. 10.1002/wsb.731

Turzańska-Pietras, K., Chachulska, J., Polechońska, L., \& Borowiec, M. (2017). Does heavy metal exposure affect the condition of Whitethroat (Sylvia communis) nestlings? Environmental Science And Pollution Research, 25 (8), 7758-7766. 10.1007/s11356-017-1064-

Vizuete, J., Pérez-López, M., Míguez-Santiyán, M. P., \& Hernández-Moreno, D. (2018). Mercury (Hg), Lead (Pb), Cadmium (Cd), Selenium (Se), and Arsenic (As) in Liver, Kidney, and Feathers of Gulls: a review. Reviews Of Environmental Contamination And Toxicology, 247 (1), 85-146. 10.1007/398_2018_16

Yang, Y., Zhang, W., Wang, S., Zhang, H., \& Zhang, Y. (2020). Response of male reproductive function to environmental heavy metal pollution in a freeliving passerine bird, Passer montanus. Science Of The Total Environment, 747, 141402. 10.1016/j.scitotenv.2020.141402 